\title{
LOGARITHMIC DERIVATIVE OF AN ENTIRE FUNCTION
}

\author{
MORRIS MARDEN ${ }^{1}$
}

\begin{abstract}
A representation for the logarithmic derivative $\left(f^{\prime} / f\right)$ of an entire function $f$ of finite order, parametrically in terms of some zeros and critical points of $f$, is derived from the Hadamard representation and applied to Lucas' type theorems and to growth estimates of $f^{\prime} / f$.
\end{abstract}

1. Introduction. In a recent paper [1] we developed a new representation for the logarithmic derivative of an entire function $f$ of finite order $\rho$. The representation features the use of some given zeros and critical points of $f$ as parameters instead of the parameters occurring in the Hadamard representation. Among the applications was the development of an analogue to Lucas' theorem that is valid for entire functions of finite order.

The present paper has as objectives:

(A) to derive our representation directly from the Hadamard one;

(B) to show by example that our entire function analogue of Lucas' theorem is sharp;

(C) to propose the relation

$$
f^{\prime}(z) / f(z)=O\left(\mid z^{\rho-1+\epsilon}\right), \quad \epsilon>0,
$$

as $|z| \rightarrow \infty$ along a suitable path or sequence of points, and to state some conditions on the growth of the zeros of $f$ sufficient to have relation (1.1) follow from our representation.

2. The representation. This is embodied in the following theorem.

THEOREM 1. Let $f$, an entire function of finite order $\rho$, have the zeros $a_{1}, a_{2}, a_{3}, \cdots$. Let $[\rho]$ be the largest integer not exceeding $\rho$ and $m a$ nonnegative integer. Let $\zeta_{1}, \zeta_{2}, \cdots, \zeta_{n}$ be any $n=m+[\rho]$ distinct zeros of the derivative $f^{\prime}$ of $f$. Then for all $z \neq a_{j}, j=1,2,3, \cdots$,

$$
f^{\prime}(z)=\frac{f(z) \Psi(z)}{\Phi(z)} \sum_{j=m+1}^{\infty} \frac{\Phi\left(a_{j}\right)}{\Psi\left(a_{j}\right)\left(z-a_{j}\right)}
$$

Presented to the Society June 4, 1969; received by the editors June 4, 1969 and, in revised form, June 24, 1970.

AMS 1970 subject classifications. Primary 30A62, 30A64; Secondary 30A08.

Key words and phrases. Hadamard representation, Lucas' theorem, growth order of logarithmic derivative, Lagrange interpolation formula.

${ }^{1}$ Work on this paper was done under N.S.F. Grants GP-11407 and GP-19615. 
where

$$
\Psi(z)=\prod_{k=1}^{n}\left(z-\zeta_{k}\right), \quad \Phi(z)=\prod_{k=1}^{m}\left(z-a_{k}\right),
$$

unless $m=0$ when $\Phi(z) \equiv 1$ and where $\Psi\left(a_{j}\right) \neq 0, j=1,2, \cdots$

Proof. Starting with the Hadamard representation (for simplicity, omitting any zero at $z=0$ ),

$$
f(z)=\Phi(z) e^{Q(z)} \prod_{j=m+1}^{\infty} E\left(z / a_{j}, p\right)
$$

where $Q(z)$ is a polynomial of degree $q \leqq \rho$, and

$$
E(u, p)=(1-u) \exp \left[u+(1 / 2) u^{2}+\cdots+(1 / p) u^{p}\right]
$$

with $p \leqq \rho$, we find that

$$
\frac{f^{\prime}(z)}{f(z)}=\frac{\Phi^{\prime}(z)}{\Phi(z)}+Q^{\prime}(z)+\sum_{j=m+1}^{\infty} \frac{z^{p}}{a_{j}^{p}\left(z-a_{j}\right)} .
$$

We may rewrite (2.2) as

$$
\Phi(z) \frac{f^{\prime}(z)}{f(z)}=g(z)+h(z)
$$

where

$$
\begin{aligned}
& g(z)=\Phi^{\prime}(z)+Q^{\prime}(z) \Phi(z), \\
& h(z)=\sum_{j=m+1}^{\infty} \frac{z^{p} \Phi(z)}{a_{j}^{p}\left(z-a_{j}\right)} .
\end{aligned}
$$

Polynomial $g(z)$ is of degree $m+q-1 \leqq n-1$. Since the $\zeta_{k}$ are zeros of $f^{\prime}$, we obtain from $(2.3)$

$$
g\left(\zeta_{k}\right)=-h\left(\zeta_{k}\right), \quad k=1,2, \cdots, n .
$$

Now, from the Lagrange interpolation formula we learn that

$$
\begin{aligned}
\frac{g(z)}{\Psi(z)} & =\sum_{k=1}^{n} \frac{g\left(\zeta_{k}\right)}{\Psi^{\prime}\left(\zeta_{k}\right)\left(z-\zeta_{k}\right)} \\
& =-\sum_{k=1}^{n} \frac{h\left(\zeta_{k}\right)}{\Psi^{\prime}\left(\zeta_{k}\right)\left(z-\zeta_{k}\right)} \\
& =-\sum_{k=1}^{n} \sum_{j=m+1}^{\infty} \frac{\zeta_{k}^{p} \Phi\left(\zeta_{k}\right)}{a_{j}^{p}\left(\zeta_{k}-a_{j}\right)\left(z-\zeta_{k}\right)} .
\end{aligned}
$$


Since in (2.2) the series is uniformly and absolutely convergent on any compact set excluding the $a_{j}$, we may interchange the sums and write

$$
\frac{g(z)}{\Psi(z)}=-\sum_{j=m+1}^{\infty} \sum_{k=1}^{n} \frac{\zeta_{k}^{p} \Phi\left(\zeta_{k}\right)}{a_{j}^{p}\left(z-a_{j}\right)}\left[\frac{1}{z-\zeta_{k}}+\frac{1}{\zeta_{k}-a_{j}}\right] .
$$

Thus,

$$
\frac{g(z)}{\Psi(z)}=\sum_{j=m+1}^{\infty} \frac{1}{a_{j}^{p}\left(z-a_{j}\right)}\left\{\frac{s\left(a_{j}\right)}{\Psi\left(a_{j}\right)}-\frac{s(z)}{\Psi(z)}\right\}
$$

where

$$
s(z)=\Psi(z) \sum_{k=1}^{n} \frac{\zeta_{k}^{p} \Phi\left(\zeta_{k}\right)}{\Psi^{\prime}\left(\zeta_{k}\right)\left(z-\zeta_{k}\right)} .
$$

For polynomial $s(z)$ we have deg $s(z) \leqq n-1$. Since (2.8) is again the Lagrange interpolation formula, we see that $s\left(\zeta_{k}\right)=\zeta_{k}^{p} \Phi\left(\zeta_{k}\right)$.

Let us define

$$
\chi(z)=z^{p} \Phi(z)-s(z),
$$

a polynomial such that $\operatorname{deg} \chi(z) \leqq p+m \leqq[\rho]+m=n$.

Since $\chi\left(\zeta_{k}\right)=0$ for $k=1,2, \cdots, n$, we infer that deg $\chi(z)=n$ and in fact that

$$
\chi(z)=c \Psi(z), \quad c=\text { constant } \neq 0 .
$$

Consequently, from (2.9) and (2.10), $s(z) / \Psi(z)=\left[z^{p} \Phi(z) / \Psi(z)\right]-c$ and thus (2.7) becomes

$$
\frac{g(z)}{\Psi(z)}=\sum_{j=m+1}^{\infty} \frac{\Phi\left(a_{j}\right)}{\Psi\left(a_{j}\right)\left(z-a_{j}\right)}-\sum_{j=m+1}^{\infty} \frac{\Phi(z) z^{p}}{\Psi(z)\left(z-a_{j}\right) a_{j}^{p}}
$$

Substituting from (2.11) and (2.5) into (2.3), we obtain

$$
\Phi(z) \frac{f^{\prime}(z)}{f(z)}=\sum_{j=m+1}^{\infty} \frac{\Phi\left(a_{j}\right) \Psi(z)}{\Psi\left(a_{j}\right)\left(z-a_{j}\right)}
$$

which completes the proof of Theorem 2 .

3. Sharpness of entire function analogue to Lucas' theorem. The Lucas theorem referred to is the one which states that the zeros of the derivative of a polynomial $f$ lie in the convex hull of the zeros of $f$. The following analogous result had been deduced (see [1]) from Theorem 1. The statement uses the notation $\mathrm{e} T$ for the complement 
of set $T, \mathfrak{F C} T$ for the (closed) convex hull of set $T$, and $\varsigma_{\gamma} T$ for the set of points from which $\mathfrak{H C} T$ subtends an angle of at least $\boldsymbol{\gamma}, 0<\boldsymbol{\gamma} \leqq \pi$.

TheOREM 2. If $\operatorname{e} \mathfrak{F C} T \varnothing \varnothing$ and $a_{j} \in T$ for $j>m$ and, when $m>0$, if also $a_{j} \in \mathrm{eS}_{\beta} T$ for $0<j<m$ and $0<m \beta<\pi$, then $f^{\prime}$ has at most $n=m$ $+[\rho]$ zeros in $\mathrm{eS}_{\gamma} T$ where $\gamma=(\pi-m \beta) /(n+1)$.

Knowing Lucas' theorem to be sharp, we now show that similarly Theorem 2 is the best possible result at least in the case $m=0$, $\rho=[\rho]=n$ (an integer). In this case, Theorem 2 asserts that, if $a_{j} \in T$ for all $j$, then at most $n$ zeros of $f^{\prime}$ lie in $\operatorname{es}_{\gamma} T$ with $\gamma=\pi /(n+1)$.

Let us on the contrary assume that this property is valid for a smaller domain $K$ contained in $\mathcal{S}_{\gamma} T, T \subset K \subset \mathcal{S}_{\gamma} T, 0<\gamma<\pi$, and that $\zeta$ is a point on the boundary $S_{1}$ of $S_{\gamma} T$ but not on the boundary $K_{1}$ of $K$. Corresponding to $\zeta$ as a point of $S_{1}$ there are points $t_{1}, t_{2} \in T$ such that

$$
\arg \left(\zeta-t_{1}\right) /\left(\zeta-t_{2}\right)=\pi /(n+1) .
$$

Let us choose a closed disk $\delta$ about $\zeta$ such that $\delta \cap K=\varnothing$.

Let us now define $\psi$ by the formula

$$
\begin{array}{r}
\log \Psi(z)=\int_{\zeta}^{z} \int_{\zeta}^{v_{n}} \cdots \int_{\zeta}^{v_{2}} \int_{\zeta}^{v_{1}}\left[\frac{\mu_{1}}{\left(v_{0}-t_{1}\right)^{n+1}}+\frac{\mu_{2}}{\left(v_{0}-t_{2}\right)^{n+1}}\right] \\
d v_{0} d v_{1} \cdots d v_{n}
\end{array}
$$

where

$$
\mu_{1}=\left|\zeta-t_{1}\right|^{n+1}, \quad \mu_{2}=\left|\zeta-t_{2}\right|^{n+1},
$$

and where $z \in \delta$ and $v_{k} \in \delta, k=0,1, \cdots, n$. Since

$$
\log \Psi(z)=\left[(-1)^{n} / n !\right] \sum_{k=1}^{2} \mu_{k} \log \left[\left(z-t_{k}\right) /\left(\zeta-t_{k}\right)\right]+G(z)
$$

where $G$ is a polynomial of degree $n$, clearly $\log \Psi(z)$ and $\Psi(z)$ are holomorphic for $z \in \delta$.

Let us now approximate to each $\mu_{k}$ by a sequence of rational numbers $\nu_{k m}$ such that for a given $\epsilon>0,0 \leqq \mu_{k}-\nu_{k m}<\epsilon / 2^{m}, k=1,2$; $m=1,2, \cdots$. If we let

$$
\log \Psi_{m}(z)=\left[(-1)^{n} / n !\right] \sum_{k=1}^{2} \nu_{k m} \log \left[\left(z-t_{k}\right) /\left(\zeta-t_{k}\right)\right]+G(z)
$$

and let $M_{k}=\max _{z \in \delta}\left|\log \left[\left(z-t_{k}\right) /\left(\zeta-t_{k}\right)\right]\right|$, then

$$
\left|\log \Psi(z)-\log \Psi_{m}(z)\right| \leqq(1 / n !)\left(M_{1}+M_{2}\right) \epsilon / 2^{m} \quad \text { for } z \in \delta .
$$


That is, the sequence $\left\{\log \Psi_{m}(z)\right\}$ converges uniformly to $\log \Psi(z)$ on $\delta$.

On the other hand, we learn from (3.2) that

$$
\Psi^{\prime}(\zeta)=\Psi^{\prime \prime}(\zeta)=\cdots=\Psi^{(n)}(\zeta)=0 .
$$

Furthermore, we see that

$$
\Psi^{(n+1)}(\zeta)=\frac{\mu_{1}}{\left(\zeta-t_{1}\right)^{n+1}}+\frac{\mu_{2}}{\left(\zeta-t_{2}\right)^{n+1}}=0
$$

due to (3.1) and the expressions $\left(\zeta-t_{1}\right)^{n+1}=\mu_{1} e^{i \omega},\left(\zeta-t_{2}\right)^{n+1}$ $=\mu 2 e^{i(\omega+\pi)}$. That is to say, $\Psi^{\prime}(z)$ has a zero of multiplicity at least $n+1$ at $\zeta$. We now conclude from Hurwitz' theorem that for sufficiently large $m$ all $\Psi_{m}^{\prime}(z)$ have at least $n+1$ zeros (counted with multiplicity) in $\delta$. The same is true of the first derivative of $\Phi_{m}(z)$ where

$$
\log \Phi_{m}(z)=(-1)^{n} n ! d_{m} \log \Psi_{m}(z)
$$

and $d_{m}$ is the least common denominator of $\nu_{1 m}$ and $\nu_{2 m}$.

However, from (3.4) and (3.5), we obtain the form

$$
\Phi_{m}(z)=e^{Q(z)}\left(z-t_{1}\right)^{\lambda_{1}}\left(z-t_{2}\right)^{\lambda_{2}}
$$

where $\lambda_{k}=d_{m} \alpha_{k m}$ and where $Q(z)$ is a polynomial of degree $n$. Hence, $\Phi_{m}(z)$, an entire function of order $n$, has its zeros on $T$ but its derivative has at least $n+1$ zeros outside the domain $K$. This contradicts the assumption that in the case $m=0, \rho=[\rho]=n$, the domain $\delta_{\gamma} T$ can be replaced by a smaller domain $K$ containing $T$ and thus verifies that Theorem 2 is sharp.

4. Growth order of logarithmic derivative. If $f$ is an entire function of order $\rho$ but with only a finite number of zeros, it is easy to verify that its logarithmic derivative satisfies relation (1.1). The relation (1.1) appears to be valid also for more general entire functions of order $\rho$, outside certain neighborhoods of the zeros of $f$. So far we have succeeded in proving, by application of Theorem 1 , the following restricted theorem.

THEOREM 3. Let $f$, an entire function of finite order $\rho$, have the zeros $a_{j}$ with $0<\left|a_{1}\right|<\left|a_{2}\right|<\cdots$ such that there exist real constants $\alpha$ and $\beta$, with $0<\beta<1<\alpha$, and with

$$
\alpha\left|a_{j}\right| \leqq \beta\left|a_{j+1}\right|, \quad j=m+1, m+2, \cdots .
$$

Let $\left\{R_{j}\right\}$ be a sequence chosen so that 


$$
\alpha\left|a_{j}\right| \leqq R_{j} \leqq \beta\left|a_{j+1}\right|, \quad j=m+1, m+2, \cdots .
$$

Then for $k$ sufficiently large

$$
f^{\prime}\left(R_{k} e^{i \theta}\right) / f\left(R_{k} e^{i \theta}\right)=O\left(R_{k}^{\rho-1+\epsilon}\right), \quad \epsilon>0 .
$$

A sufficient condition for (4.1) is that, as $j \rightarrow \infty$,

$$
\lim \sup \left(\left|a_{j}\right| /\left|a_{j+1}\right|\right)=\mu
$$

where $0<\mu<1$. For, given any $\nu$ with $\mu<\nu<1$, we can find $m$ so that $\left|a_{j}\right| /\left|a_{j+1}\right|<\nu$ for $j>m$ and thus can choose $\alpha=\nu^{-1 / 2}$ and $\beta=\nu^{1 / 2}$.

\section{REFERENCES}

1. M. Marden, On the derivative of an entire function, Proc. Amer. Math. Soc. 19 (1968), 1045-1051. MR 38 \#322.

University of Wisconsin-Milwaukee, Milwaukee, Wisconsin 53201 